\title{
A Systematic Review of Interventions Used to Increase Blood Donor Compliance with Deferral Criteria
}

\author{
Julia C. Cutts ${ }^{a}$ Brendan Quinn ${ }^{a, c}$ Clive R. Seed $^{b}$ George Kotsiou $^{b}$ \\ Ruth Pearson ${ }^{\mathrm{a}}$ Nick Scott $^{\mathrm{a}, \mathrm{c}}$ David P. Wilson ${ }^{\mathrm{a}}$ Mary Ellen Harrod ${ }^{\mathrm{d}}$ \\ Lisa Maher ${ }^{\mathrm{a}}$ e Sharon Caris ${ }^{f}$ Alex J. Thompson ${ }^{g}$ Michael Farrell ${ }^{\text {h }}$ \\ Joanne Pink ${ }^{b}$ Margaret E. Hellard ${ }^{a, c, i, j}$
}

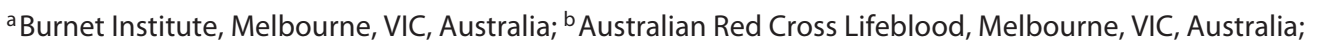
'Department of Epidemiology and Preventive Medicine, Monash University, Melbourne, VIC, Australia; ${ }^{\mathrm{d}}$ NSW Users and AIDS Association, Surry Hills, NSW, Australia; ${ }^{\mathrm{E}}$ Kirby Institute, UNSW Sydney, Sydney, NSW, Australia; ${ }^{f}$ Haemophilia Foundation Australia, Malvern East, VIC, Australia; ${ }^{9}$ Department of Gastroenterology, St Vincent's Hospital and the University of Melbourne, Melbourne, VIC, Australia; ${ }^{\mathrm{h}}$ National Drug and Alcohol Research Centre, University of New South Wales, Sydney, NSW, Australia; 'Department of Infectious Diseases, The Alfred Hospital, Melbourne, VIC, Australia; ${ }^{j}$ Doherty Institute and School of Population and Global Health, University of Melbourne, Melbourne, VIC, Australia

\section{Keywords}

Blood donors · Deferral · Compliance · Screening

\begin{abstract}
Background and Objectives: Pre-donation screening of potential blood donors is critical for ensuring the safety of the donor blood supply, and donor deferral as a result of risk factors is practised worldwide. This systematic review was conducted in the context of an expert review convened by the Australian Red Cross Lifeblood in 2013 to consider Lifeblood's injecting drug use (IDU)-related policies and aimed to identify studies assessing interventions to improve compliance with deferral criteria in blood donation settings. Materials and Methods: MEDLINE/PubMed, OVID Medline, OVID Embase, LILACS, and the Cochrane Library (CENTRAL and DARE) databases were searched for studies conducted within blood donation settings that examined interventions to increase blood donor compliance with deferral criteria. Observational and experimental studies from all geographical areas were considered. Results: Ten studies were identified that tested at least one intervention to improve blood donor compliance with deferral criteria, including comput-
\end{abstract}

erized interviews or questionnaires, direct and indirect oral questioning, educational materials, and a combination of a tickbox questionnaire and a personal donor interview. Highquality evidence from a single study was provided for the effectiveness of a computerized interview in improving detection of HIV risk behaviour. Low-quality evidence for the effectiveness of computerized interviews was provided by 3 additional studies. Two studies reported a moderate effect of direct questioning in increasing donor deferral, but the quality of the evidence was low. Conclusion: This review identified several interventions to improve donor compliance that have been tested in blood donation settings and provided evidence for the effectiveness of computerized interviews in improving detection of risk factors.

(C) 2020 S. Karger AG, Basel

\section{Introduction}

To reduce the risk of transfusion-transmissible infections (TTI), the Australian Red Cross Lifeblood (formerly the Australian Red Cross Blood Service; hereafter: "Lifeblood") maintains a policy of exclusion or deferral of 
potential blood donors as a result of "group" risk. Because illicit injecting drug use (IDU) is a key transmission route of various TTI, particularly hepatitis C virus [1], until September 2018, potential blood donors in Australia who reported a history of IDU were deferred indefinitely (permanently excluded). In addition, because TTI such as hepatitis B and HIV, and in some circumstances, hepatitis C, can be sexually transmitted [2], potential donors are deferred for 12 months if they report having engaged in sexual activity in the past year with someone who may have injected drugs. These recommendations were not supported by high-quality evidence, and the system is predicated on individuals self-reporting risk behaviour in blood donation settings.

In late 2013, Lifeblood convened an Expert Review Committee to consider Lifeblood's IDU-related guidelines and to recommend how exclusions or deferrals from donation should be structured. The Expert Review Committee's 2015 report to Lifeblood recommended that no changes be made to the guidelines due to a lack of data and evidence related to IDU risk and blood donations [3], and that further information and data be obtained to inform the Committee's future considerations. One recommendation was that a systematic review be undertaken to identify and examine the existing evidence for strategies to increase compliance with deferral criteria in blood donation settings worldwide, which we report herein. The other key recommendations were the collection of data on the prevalence of lifetime blood donation among Australian PWID (people who inject drugs), and that these data be used to inform a mathematical model to estimate the additional risk to Australia's blood supply associated with changing Lifeblood's IDU-related deferral period to 1 or 5 years since the last injection episode [4]. The Expert Review Committee reconvened in 2017 to discuss the findings of the systematic review and the results of mathematical modelling, and ultimately recommended that the IDU-related deferral period be changed from permanent exclusion to a 5-year deferral from a PWID's last injecting episode. Lifeblood subsequently made a successful submission to Australia's blood sector regulator (Therapeutic Goods Administration) to change the deferral period from indefinite to 5 years since the last injecting episode, and this policy came into effect in September 2018.

Before every donation in Australia, potential donors complete a donor health questionnaire containing questions about medical and lifestyle factors associated with an increased TTI risk. The donor health questionnaire is reviewed in a private and confidential face-to-face interview with the donor, and a legally binding declaration is signed. Blood safety depends upon the donor's complete and truthful answers to all pre-donation screening questions assessing TTI risk/exposure (termed "compliance").
An online survey of TTI test-negative Australian blood donors found that $1.65 \%$ were non-compliant (that is, failed to disclose at donation something that would lead to deferral if disclosed) with at least one screening question [5]. A history of IDU was reported by $0.36 \%$ of participants, indicating non-compliance with Lifeblood's main IDU-related donor screening question [5]. This level of non-compliance is mid-range compared to similar studies conducted in Canada (0.15\%) [6] and the United States (0.51\%) [7]. About 3-quarters of Australian respondents with a history of IDU reported that their most recent injecting episode occurred more than 5 years before their last donation [5].

Possible reasons for non-compliance with deferral criteria include discomfort at raising questions with blood centre staff, a perceived lack of privacy, fear of embarrassment, a perception that questions are overly personal [5, $6,8]$, and failure of the donor to carefully read instructions or a lack of comprehension of the information presented to them [8-10]. In some cases, potential donors may believe that they are healthy, and that their behaviour does not place them at risk of transmitting infection $[6,8$, $9,11,12]$.

In some contexts, potential donors present to blood banks to receive free HIV testing and assume that infections will be detected, and that it is therefore not necessary to answer screening questions honestly $[5,8,11,13-16]$. Albeit at a low level internationally and despite the availability of free testing of sexually transmitted infections via other services, test seeking was found to be associated with non-compliance among Australian male donors [5]. Another hypothesised reason for non-compliance is the belief that the policy of deferring certain groups (e.g., men who have sex with men) is unfair, and that protest in the form of non-compliance is acceptable $[16,17]$.

Included in this systematic review are studies that investigated the effectiveness of interventions or procedure changes in blood donation settings on outcomes including donor deferral, disclosure of risk factors, and rates of errors and omissions. Study participants included prospective blood donors attending blood donation sites in any geographical location worldwide, and both observational and experimental study designs were included. The overall objective of this review was to provide an evidence base to inform strategies to improve blood donor compliance with donor selection criteria particularly in the context of an expert review of Lifeblood's IDU-related guidelines.

\section{Materials and Methods}

\section{Review Protocol}

The Preferred Reporting Items for Systematic Reviews and Meta-Analyses (PRISMA) specifications were adhered to in the conducting and reporting of this systematic review and meta-analysis 


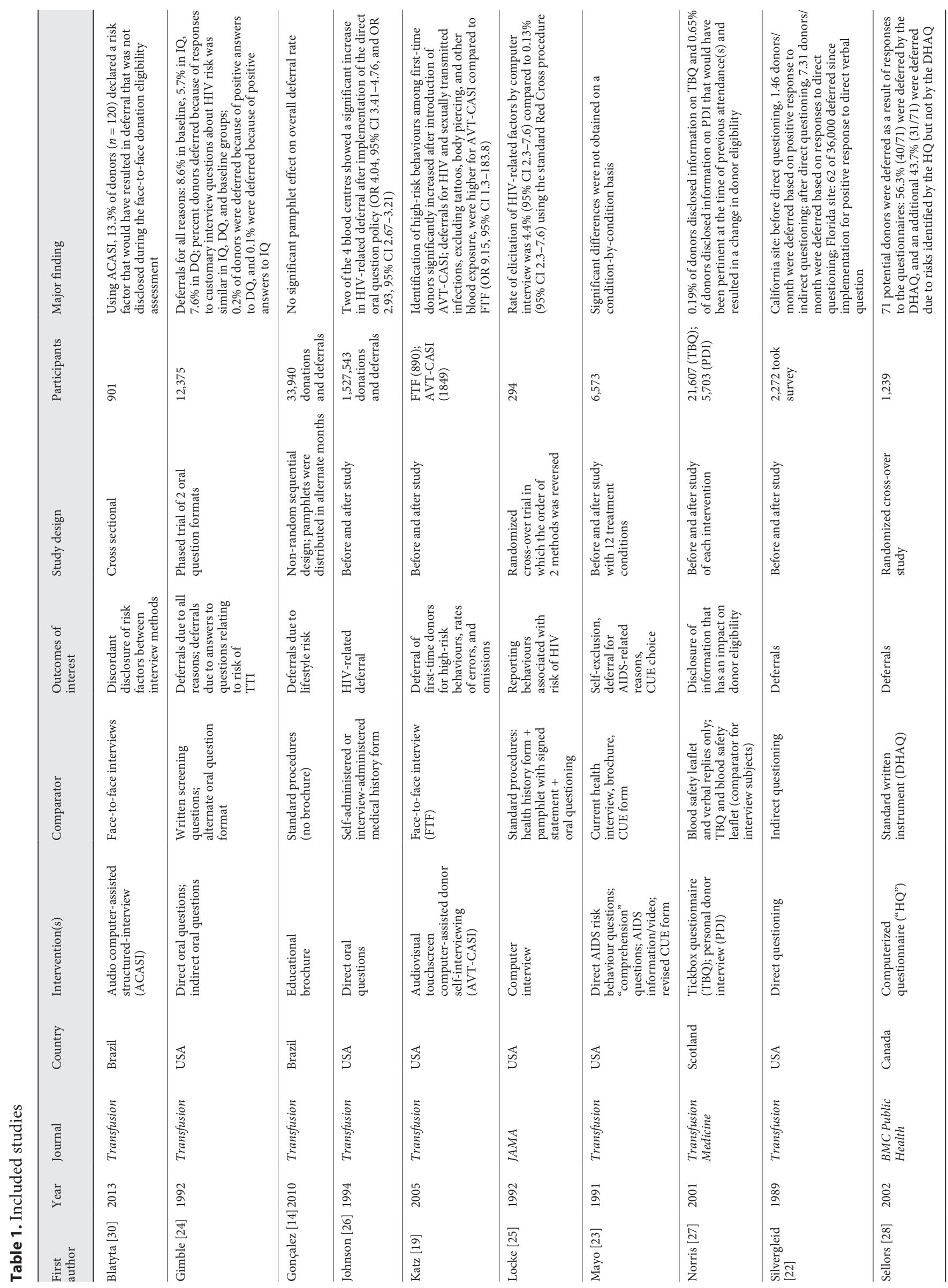




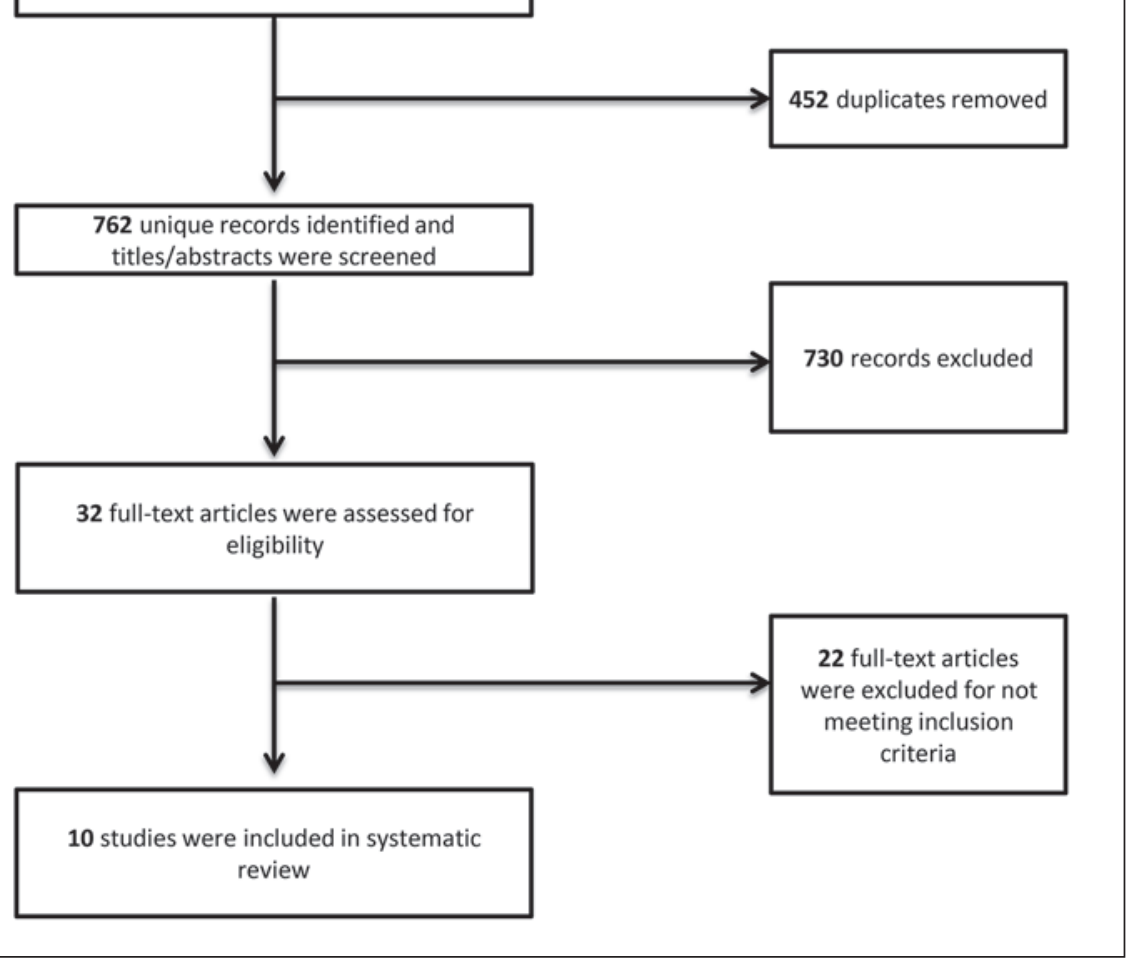

[18]. A completed PRISMA checklist is included in online suppl. file 1 (see www.karger.com/doi/10.1159/000509027 for all online suppl. material).

Search Methods for the Identification of Studies

MEDLINE/PubMed, OVID Medline, OVID Embase, LILACS, and the Cochrane Library (CENTRAL and DARE) databases were searched for studies published in all years up to and including August 16, 2019, that examined interventions used to increase blood donor compliance with deferral criteria. Where possible, searches were limited to research involving humans. The full search strategy for one database (PubMed) is provided (online suppl. file 2). Citation titles and abstracts were screened for relevance, and publications identified as potentially relevant were retrieved in full text. Full-text articles were examined to determine whether they met the eligibility criteria as outlined below. The reference lists of obtained studies were searched for additional relevant studies. Studies reported in languages other than English were included and translated into English using online translation applications. Leveraging a number of international alliance partnerships, unpublished data were requested from international blood services.

\section{Eligibility Criteria}

Observational (prospective and retrospective cohorts, crosssectional surveys and case-controlled studies) and experimental studies were considered eligible if they assessed the use of interventions or changes to procedure that may increase compliance with deferral criteria in a blood donation setting. The outcomes measured in the systematic review included donor deferral due to all reasons, donor deferral due to positive answers to screening questions on TTI risk, disclosure of risk factors, and rates of errors and omissions. For the purpose of this review, compliance was defined as the donor's full and frank disclosure in response to questions posed during pre-donation screening. Studies from all geographical locations were included.

\section{Risk of Bias in Individual Studies}

At an individual study level, selection bias was assessed to determine whether participants were representative of the general (donor) population by reviewing individual study inclusion and exclusion criteria. Risk of bias and overall quality of the evidence included in this review was assessed using GRADEpro GDT software to employ the Grading of Recommendations, Assessment, Development, and Evaluation (GRADE) approach [19-21], and results were presented in evidence tables according to intervention and outcome. The limited number of studies examining any one outcome precluded risk of bias analysis across studies.

Data Analysis

Where available, measures of association (odds ratios [OR], risk ratios) and their $95 \%$ confidence intervals [CI]) were extracted or derived using data reported in the publications. Where a study did not provide measures of association (or they could not be calculated with the information provided), the study results were presented as they were in the publication. The findings of individual studies were summarized in a word document, and key characteristics of each study were recorded in a table (Table 1).

\section{Results}

\section{Identification of Studies}

Database searches identified a total of 762 unique records, from which 32 potentially relevant studies were 
identified based on their abstracts (Fig. 1). Examination of the full texts of these studies identified 10 studies that tested at least 1 intervention designed to improve blood donor compliance with deferral criteria in a blood donation setting (Table 1) [14, 22-30]. The studies were conducted in the United States [22-26, 29], Brazil [14, 30], Scotland [27], and Canada [28], and published between 1989 [22] and 2013 [30]. Study designs included a crosssectional study [30], a non-random sequential design study [14], a phased trial [24], before-and-after studies $[22,26,27,29]$, randomized cross-over trials $[25,28]$, and 1 study that tested 12 "treatment conditions" [23]. The most commonly examined outcome was deferral of donors.

\section{Description of Included Studies}

Computerized Interviews and Questionnaires

The use of a computerized interview or questionnaire in blood donation settings was examined in 4 studies (Table 2) [25, 28-30]. Audio computer-assisted self-interview (ACASI) is a software system designed to deliver complex interviews/questionnaires in a standardised, multilingual, audio format. Typically, respondents listen to recorded questions through headphones whilst viewing them on a computer screen; answers are entered using the computer keyboard or touchscreen, and are recorded without the participation of an interviewer [31]. ACASI has been shown to reduce social desirability bias and increase disclosure of socially sensitive behaviours, including injection drug use [32].

Blatyta et al. [30] performed a cross-sectional study of 901 HIV-negative, eligible blood donors at 4 large Brazilian blood centres to determine disclosure of risk factors using ACASI compared to face-to-face interviews. Using ACASI, 120 (13.3\%) donors declared a risk factor for HIV, including IDU, that would have resulted in deferral that was not disclosed during a preceding face-to-face interview [30]. Of these, only 2 donors $(0.2 \%)$ reported using intravenous drugs, and $2(0.2 \%)$ were partners of intravenous drug users. This study provided evidence that donors preferred to answer stigmatizing and socially sensitive questions in the relative privacy accorded by ACASI. Limitations of the study included the fact that all donors completed the face-to-face interview followed by the ACASI, precluding investigation of the effect of the order of the interview method, and that the ACASI contained a greater number and more detailed questions than the face-to-face interview [30].

Katz et al. [29] described a study conducted in a blood centre in Iowa, USA, in which deferral rates for first-time blood donors were compared between 2 periods: the 12 months preceding the installation of an audio-visual touchscreen computer-assisted donor self-interviewing system (AVT-CASI), when donors were interviewed face 
to face ( 890 donors), and in the 18 months after introduction of the system (1,849 donors). The use of AVT-CASI was associated with a 9-fold increase in the odds of disclosure of socially sensitive or stigmatizing behaviours (OR 9.15, 95\% CI 1.3-183.8) and a significant decline in the rate of errors and omissions (OR 0.39; 95\% CI 0.240.63) [29].

In a randomized cross-over trial conducted at 2 sites in the USA, Locke et al. [25] compared the rate at which HIV-related risk factors were elicited by a newly designed computer interview compared to standard American Red Cross procedures, including a health history form, a pamphlet with a signed statement, and oral questioning. The rate of elicitation of HIV-related risk factors by computer interview was $4.4 \%$ (95\% CI 2.3-7.6) compared with $0.13 \%$ (95\% confidence upper bound 0.28 ) using the standard procedures. Importantly, this study investigated the order of administration of the interview method and found it had no effect on detection of high-risk individuals or donors' reactions to a computer interview [25].

In a randomized cross-over trial of blood donors $(n=$ $1,239)$ at 133 Canadian clinics, Sellors et al. [28] compared deferral rates associated with the use of a computerized handheld device (HealthQuiz or "HQ") to those resulting from the standard written questionnaire (Donor Health Assessment Questionnaire, DHAQ). Prospective donors were randomly selected to participate in the study and were randomly assigned to receive either the HQ followed by the DHAQ or the DHAQ followed by the HQ. The HQ questions were based on the standard written DHAQ, with questions appearing on the screen one at a time. In contrast, the DHAQ was a self-administered list of questions with "yes" and "no" check boxes. Together the 2 questionnaires led to the deferral of $5.7 \%$ of donors: $3.2 \%$ were deferred on the basis of answers to the DHAQ reviewed by clinic staff, and an additional 2.5\% were identified by items on the HQ which were not identified on the DHAQ [28]. There were some cases where risks were identified on DHAQ and denied on HQ, but the total numbers (if any) deferred based on questions identified solely on DHAQ were not reported. The authors noted that a limitation of the study was that the majority of the participants were repeat donors and were therefore familiar with the standard instrument and may not have read the questions carefully [28].

Each of the 4 published studies that investigated selfcompleted computer-based questionnaires/interviews as an intervention to improve donor compliance demonstrated that the technology increased donor disclosure of risk factors.

Direct Oral Questions

In the early 1990s, many blood centres in the USA implemented procedural changes in response to revised
Food and Drug Administration (FDA) recommendations for screening prospective donors at risk of HIV. One such recommendation was that donors should be asked direct health history questions about behaviours that put them at risk of HIV, and, preferably, these questions should be asked orally $[33,34]$. Direct or explicit questions ask about the individual's own behaviour, for example "have you ever injected non-prescribed drugs, even once?" In contrast, indirect questions are generally comprehension or belief-based, for example "Do you understand that you should not give blood if you have ever injected non-prescribed drugs, even once?"

In a before-and-after study, Silvergleid et al. [22] assessed the impact of explicit questions about high-risk behaviours on donor attitudes and deferral patterns at community blood centres in California and Florida.

Prior to the implementation of direct questioning, donors were requested to read a brochure defining "highrisk groups" and were then asked, in writing, to defer themselves if they belonged to any of the described groups (indirect questioning). In the period preceding the introduction of direct questions at the California site, 1.46 donors were deferred per month based on responses to indirect questions. In the period following the introduction of direct questioning, 7.31 donors were deferred per month based on responses to direct questions. At the Florida site, following implementation of direct questioning, 62 of 36,000 potential donors were deferred due to at least 1 positive response to direct verbal questions, but a comparison with deferral numbers prior to the introduction of the new policy was not presented [22].

Gimble and Friedman [24] reported on the phased introduction of indirect and direct oral questions about HIV risk behaviours, in addition to the customary written health history interview, at 8 regional blood centres in the USA. In the indirect question group, participants were asked whether they understood the 7 ineligible donor risk behaviours; in the direct questioning group, they were asked if they had engaged in any of these behaviours. Across all the blood centres, $8.6 \%(398 / 4,572)$ of those who undertook the customary health history interview without any additional questions, 5.7\% $(175 / 3,050)$ of those asked additional indirect questions, and 7.6\% (362/4753) of those asked additional direct questions were deferred for all reasons. The percentage of donors deferred solely because of positive answers to direct questions $(0.2 \%)$ was double that deferred solely by indirect questions $(0.1 \%)$ [24].

Johnson et al. [26] performed a before-and-after study evaluating the impact of the FDA's direct oral question policy on HIV-related deferral and HIV seroprevalence at 4 blood centres in the USA. In 1990, prospective donors were asked to read written material entitled "What You Must Know before Giving Blood" and were subsequently asked 
direct questions about the symptoms of HIV infection and 1 indirect question asking whether they understood who should not donate blood. In 1991, the policy changed: donors read the same material, but were asked questions about specific HIV risk behaviours and were required to answer "yes" or "no" to each question. At each centre, the number of HIV-related deferrals per 100,000 donations increased after the introduction of direct oral questioning. The increase in deferrals was significant at 2 of the 4 centres: south-eastern Michigan: OR 4.03, 95\% CI 3.41-4.76, and Penn-Jersey: OR 2.93, 95\% CI 2.67-3.21 [26].

Mayo et al. [23] tested 5 new screening materials aimed at excluding potential donors at risk of HIV at blood collection sites in 2 eastern cities in the USA. The materials included direct risk behaviour questions; "comprehension" questions asking donors if they understood that they should not donate blood if they had engaged in specific risk behaviours; an AIDS information booklet; an AIDS information video; and a revised confidential unit exclusion (CUE) form with pictures. The study comprised 12 distinct "treatment conditions" to enable data to be collected about the effects of the new materials alone and in combination. Data were collected for 6,573 prospective donors who entered the blood collection sites in the 2 cities from November 1989 to April 1990, and the order of treatment conditions was varied between sites, with all required data collected for one condition before testing the next condition. A total of 122 individuals selfexcluded, were deferred for AIDS-related reasons, or used the CUE form to indicate that their blood should not be used. On a condition-by-condition basis, there was no significant difference in the proportion of individuals identified as high risk by these methods. However, among individuals identified as high risk, a greater proportion $55.1 \%(27 / 49)$ were deferred following the health history interview that included the direct behaviour questions, compared to $20 \%(8 / 40)$ who received the original health interview, and $27.6 \%(8 / 29)$ who received the interview with comprehension questions [23]. Participants who received the new brochure scored higher on a comprehension test than those who received the current brochure, but there was no significant increase in the number of atrisk donors who were screened out with the new brochure. Potential donors who were asked the comprehension questions as well as the health history questions scored higher on a multiple choice comprehension test than those who received the behaviour questions, but significantly more at-risk donors were screened out in the group that were asked behaviour questions in addition to the current health history questions [23].

\section{Educational Materials}

The US FDA recommends donors be provided with educational material before each donation to explain the risk of HIV transmission by blood and blood products and certain behaviours associated with increased risk of HIV [35].

As described above, Mayo et al. [23] tested the effectiveness of educational materials including an AIDS information booklet; an AIDS information video; and a revised CUE form with pictures but found no difference in the proportion of prospective donors identified as high risk between conditions.

In a study by Gonçalez et al. [14], an educational brochure was distributed to donors in alternating months at a collection centre in Sao Paulo, Brazil. The brochure was designed to increase potential donors' knowledge about screening test performance and to reduce test-seeking behaviours. The authors hypothesized that after reading pamphlets, donors who were infected with HIV or had high-risk behaviours would be more likely to self-defer from donating compared with other donors. In the 2nd half of each month, a short anonymous questionnaire about donor's knowledge of HIV infection was distributed. The pamphlet had no significant impact on the overall deferral rate, the rate of leaving without donating, or the rate of positive TTI disease markers. However, the use of the pamphlet was associated with a non-significant and slight decrease in the HIV risk deferral rate [14].

\section{Tickbox Questionnaire and Personal Donor}

Interview

Norris and Galea [27] assessed the impact of a tickbox questionnaire and a personal donor interview on donor and recipient safety in Scotland. A questionnaire introduced in 1997 contained tailored questions requiring "yes/no" responses to assess individuals' eligibility for donation. Responses to the questionnaire were reviewed by a health screener who asked a secondary set of questions about recent medical/travel history and comprehension of the questions asked. In 1998, a personal donor interview for 1st-time and 2-year lapsed donors was introduced. This interview, conducted by a nurse or doctor, enabled review of the responses to the tickbox questionnaire, followed by direct oral questions about possible risk behaviours. This study involved following individual donors prior to and after the introduction of the new methodologies and measuring instances or "hits" where the questionnaire or interview prompted a donor to divulge information which they would not otherwise have divulged, with the new information having an impact on donor eligibility. A total of 21,607 donors undertook the questionnaire, resulting in 42 "hits" (0.19\%), and 5,703 donors had a personal donor interview, resulting in 37 "hits" (0.65\%) [27].

\section{Risk of Bias Analysis}

The quality of the evidence included in this review was assessed by presenting the results in evidence tables con- 


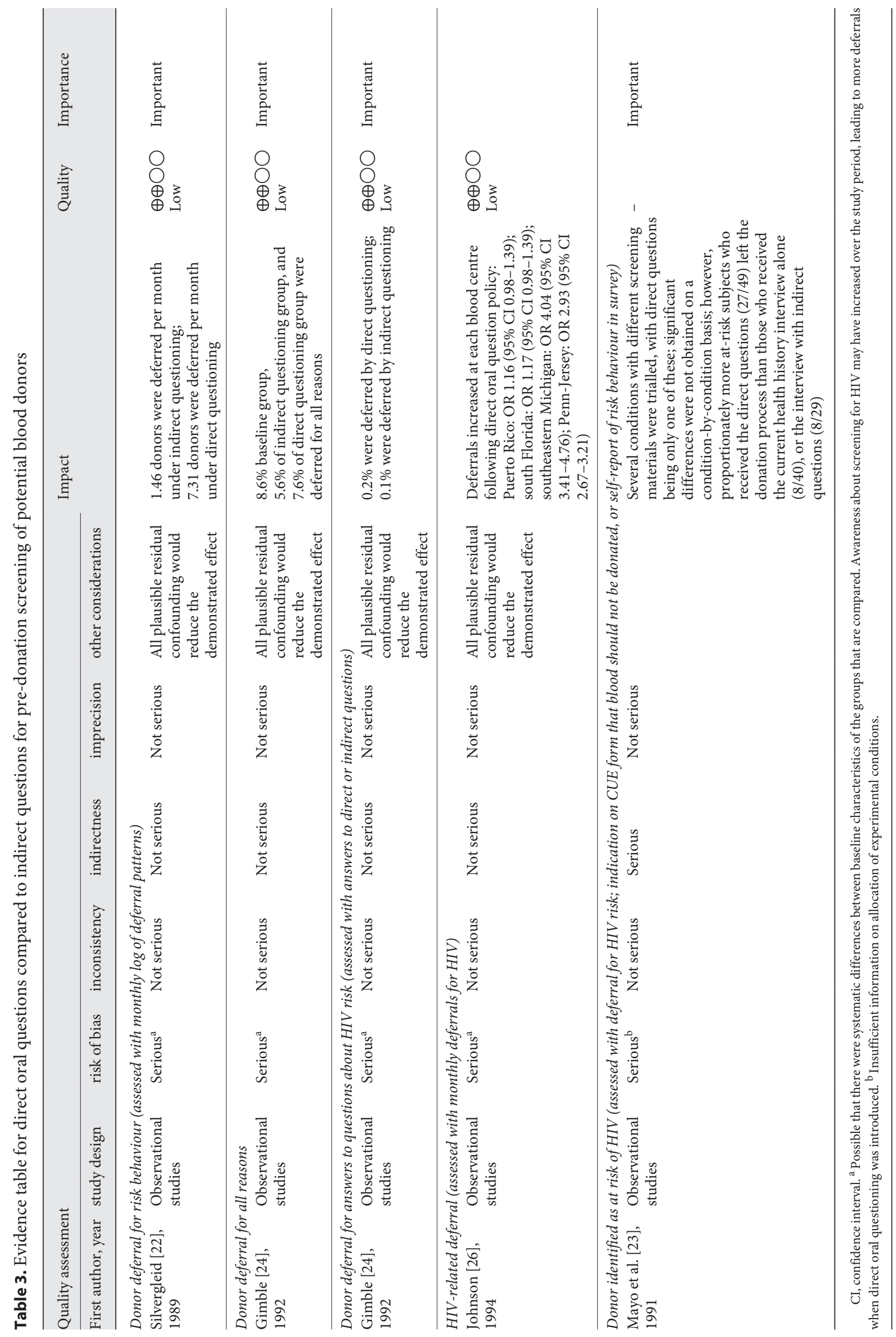



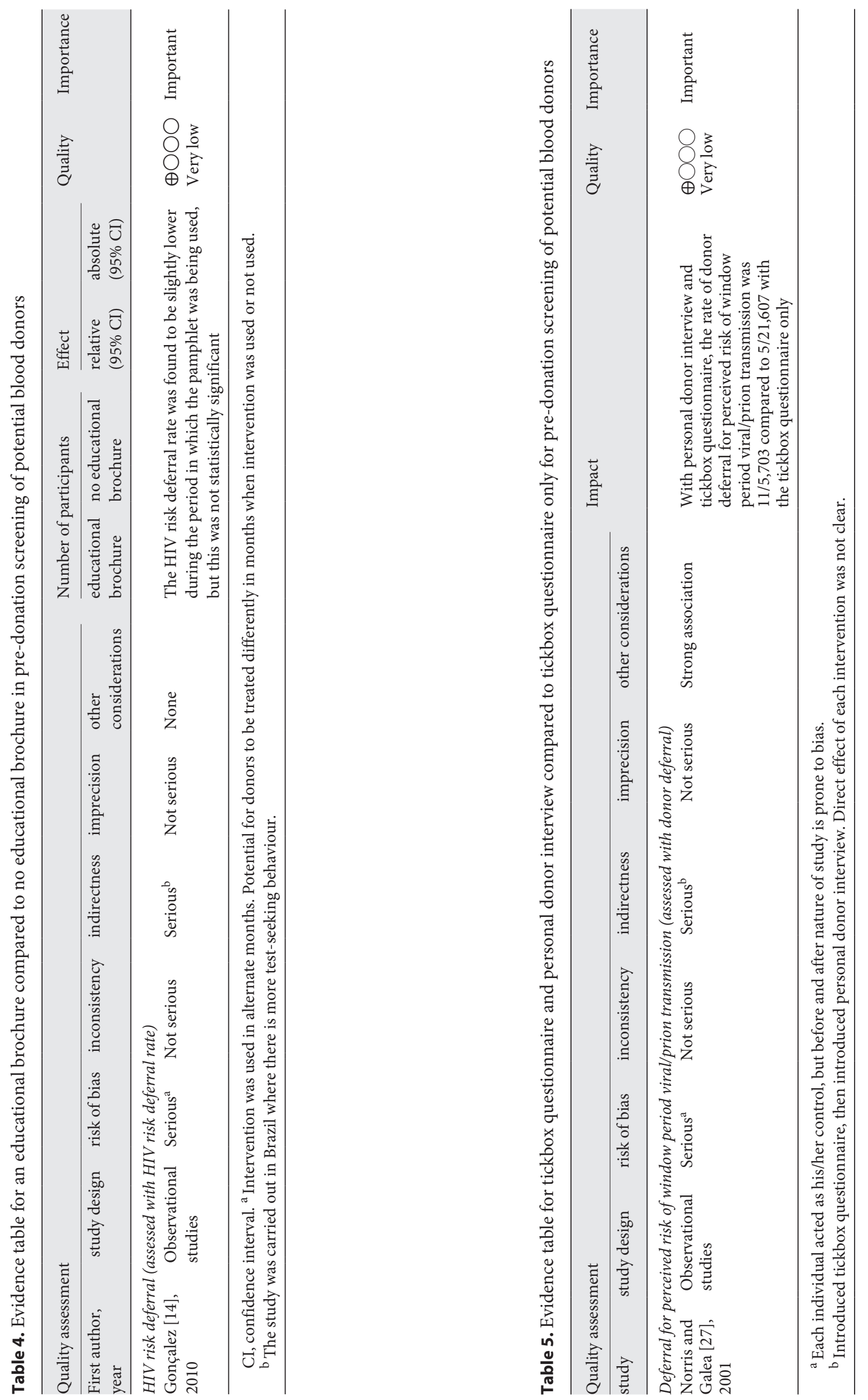
structed using GRADEpro software (Tables 2-5) [21]. Results were tabulated according to intervention (research question) and outcome. The evidence presented in Locke et al. [25] for the effectiveness of a computer-based interview compared to standard American Red Cross procedures for detecting behaviours associated with a risk of HIV acquisition, or symptoms compatible with AIDS, was deemed to be of "high" quality. This study was a randomized cross-over trial. In contrast, the evidence for all other interventions and outcomes assessed was deemed to be of low or very low quality. Many of the studies were before-and-after studies, which were uncontrolled and should be treated with caution: the absence of an appropriate comparison group means that it is impossible to determine what would have happened without the intervention [36]. Furthermore, before-and-after studies are particularly susceptible to problems with confounding and regression to the mean [36].

\section{Discussion}

This systematic review was conducted in the context of an expert review of the Australian Red Cross Lifeblood's IDU-related guidelines and was aimed at identifying interventions to improve donor compliance with deferral criteria. Included studies assessed the effectiveness of computer-based self-interviews, direct oral questioning, a tickbox questionnaire, and educational brochures. Evidence was presented that computerized interview techniques and direct oral questioning can increase the number of deferrals in blood donation settings [22, 24-26, 28-30]. Of particular note, high-quality evidence for the effectiveness of a computerized interview in increasing disclosure of HIV risk behaviours was provided by a randomized cross-over trial [25].

The findings of this systematic review support the notion that computerized interviews may improve donor compliance. Compared to traditional self-administered, pen-and-paper questionnaires, computerized interviews have been shown to increase self-reporting of illicit drug use, including injection drug use, violence, and injecting and sexual risk behaviours in a range of settings [31, 32, 37-40]. Computerized interviews can incorporate question branching, automated consistency, and range checking, and measurement and transcriptional errors are minimized [41]. Computerized interviews offer many of the advantages of face-to-face interviewing without the negative aspects of social desirability bias [42]. ACASI systems have the capacity to include an audio component to minimize the effects of literacy challenges and enhance perception of privacy, a visual component to improve comprehension [43], and a touchscreen for ease in responding to interview questions in private [29]. Anony- mous surveys of blood donors have provided further evidence for the value of computerized interviews in eliciting sensitive information: in a Canadian study, donors who had admitted to IDU reported that answering questions on a computer would help them to be more truthful [6], and in a survey of Australian donors, female donors who were noncompliant were more likely to indicate a preference for a computerized questionnaire than compliant female donors [5].

Improved educational materials may contribute to donor comprehension of TTI risk factors, but firm evidence of efficacy was lacking in the examined literature [14,23]. Interestingly, evidence suggests that while novel educational materials may increase comprehension of concepts such as "window periods" for TTI testing, this improvement in understanding may not necessarily translate to increased deferrals due to disclosure of a behavioural risk [23]. Indeed, it has been suggested that some high-risk donors will continue to donate irrespective of how much educational material is improved [44].

In addition to the adoption of computerized interviews to improve the collection of sensitive behavioural information, the application of cognitive science to improve questionnaire design may increase donor compliance with pre-donation screening. Comprehensive reviews of the Canadian and US blood donor questionnaires have identified factors that may contribute to failure to answer screening questions accurately [42, 45, 46]. False-positive and false-negative errors to individual questions can be a result of "context effects," whereby respondents tend to guess at what a question is asking, rather than answering the question literally $[42,45]$. The structure and layout of a questionnaire is important: questions should be worded as simply as possible $[42,46]$ and organized by topic within a time frame, or a time frame within a topic [42, 46, 47]. Furthermore, condensing items into a single question in the form of a list inhibits attention and recall [42], with donors being more likely to recall items that appear earlier in the list [47].

Motivations for non-compliance with deferral criteria may depend upon the geographical setting of the study site. For example, a cross-sectional study conducted in Sao Paulo, Brazil, indicated that $8.8 \%$ of donors were possible test seekers [13]. In such contexts, blood banks may be perceived as convenient settings for free HIV testing, and donors may be less likely to answer screening questions honestly $[13,14]$. It is also important to consider whether paid donation is practiced in particular settings, as this may influence donor motivations and tendency for truthfulness during pre-donation screening, although definitive evidence for an effect of incentives on blood safety is lacking [48].

Limitations of this review include the small number of studies that directly assessed the effectiveness of interven- 
tions designed to improve donor compliance, and the lack of studies focused specifically on minimizing noncompliance among current or former people who inject drugs. Measuring the relatively rare outcome of noncompliance is difficult, firstly, because there is no "gold standard" for eliciting sensitive information [23], and secondly because it is logistically difficult to conduct a randomized study with sufficient sample size to detect differences between interventions. Furthermore, trials of interventions designed to improve donor compliance in blood donation settings, rather than purely experimental settings, are constrained by the realities of the blood donation setting itself. Another limitation of this review is that the comparator, or control state, for the interventions varied between studies, precluding direct comparisons of effect sizes across studies. Furthermore, combined estimates or meta-analyses of data were not possible due to methodological heterogeneity across studies.

The substantial differences in the study years (19892013) coincides with major improvements in antiretroviral therapy for HIV, which may have changed donor perceptions of the seriousness of HIV. Furthermore, the level of general population literacy regarding TTI has most likely improved since the late 1980s, suggesting that comparison of results across studies undertaken in different decades should be treated with caution.

In conclusion, this study identified several interventions to improve donor compliance tested in blood donation settings. High-quality evidence from a single study was provided for the effectiveness of a computer interview in improving detection of HIV risk behaviours [25]. Low-quality evidence for the effectiveness of computerized interviews, including ACASI, was provided by 3 additional studies [28-30]. There was some evidence provided by 2 studies for a moderate effect of direct questioning in increasing donor deferral, but the quality of the evidence was low $[22,26]$. Further, randomized, con- trolled studies conducted in a broad range of geographical settings are necessary to establish the effectiveness of the interventions assessed in this systematic review.

\section{Statement of Ethics}

Ethics approval was not required for this study as it involved the collation of data from previously published studies. The PRISMA specifications were adhered to in the conducting and reporting of this systematic review and meta-analysis.

\section{Disclosure Statement}

M.E.H. and the Burnet Institute receive funding from Gilead Sciences, Abbvie, and GSK for investigator-initiated research. All other authors have no conflicts of interest to declare.

\section{Funding Sources}

This work was funded by the Australian Red Cross Lifeblood and conducted at the Burnet Institute, Melbourne. The Australian government funds the Australian Red Cross Lifeblood for the provision of blood, blood products, and services to the Australian community. The Burnet Institute receives operational infrastructure support from the Victorian government. Margaret E. Hellard and Lisa Maher receive support from the NHMRC as Research Fellows.

\section{Author Contributions}

C.S., G.K., R.P., N.S., D.P.W., M.E.H., L.M., S.C., A.J.T., M.F., J.P., and M.E.H. were involved in review conception and design through participation in involvement in an expert review convened by Australian Red Cross Lifeblood to consider Lifeblood's IDU-related guidelines. J.C.C., B.Q., C.S., G.K., and M.H. developed the review protocol. J.C.C. performed searches, identified publications to include in the review, synthesised results, and wrote the first draft of the review. All authors critically revised manuscript.

\section{References}

1 Pouget ER, Hagan H, Des Jarlais DC. Metaanalysis of hepatitis $\mathrm{C}$ seroconversion in relation to shared syringes and drug preparation equipment. Addiction. 2012 Jun; 107(6): 1057-65.

2 Sasadeusz J, Locarnini S, Kidd M, Bradford D, Danta M. HIV, HBV, HCV and STIs: similarities and differences. HIV, viral hepatitis and STIs: a guide for primary care. Australasian Society for HIV Medicine; 2008. pp. 9-27.

3 Quinn B, Seed C, Keller A, Maher L, Wilson $\mathrm{D}$, Farrelle $\mathrm{M}$, et al. Re-examining blood donor deferral criteria relating to injecting drug use. Int J Drug Policy. 2017 Oct;48:9-17.

4 Quinn B, Pearson R, Cutts J, Seed C, Scott N, Hoad V, et al. Blood donation amongst people who inject drugs in Australia: research sup- porting policy change. Vox Sang. 2020 Apr; 115(3):162-70.

5 Lucky TT, Seed CR, Waller D, Lee JF, McDonald $\mathrm{A}$, Wand $\mathrm{H}$, et al. Understanding noncompliance with selective donor deferral criteria for high-risk behaviors in Australian blood donors. Transfusion. 2014 Jul;54(7): 1739-49.

6 O’Brien SF, Xi G, Yi QL, Goldman M. Understanding non-disclosure of deferrable risk: a study of blood donors with a history of intravenous drug use. Transfus Med. 2010 Feb; 20(1):15-21.

7 Williams AE, Thomson RA, Schreiber GB, Watanabe K, Bethel J, Lo A, et al. Estimates of infectious disease risk factors in US blood donors. Retrovirus Epidemiology Donor Study. JAMA. 1997 Mar;277(12):967-72.
8 Orton SL, Stramer SL, Dodd RY, Alter MJ. Risk factors for HCV infection among blood donors confirmed to be positive for the presence of HCV RNA and not reactive for the presence of anti-HCV. Transfusion. 2004 Feb; 44(2):275-81.

9 Leitman SF, Klein HG, Melpolder JJ, Read EJ, Esteban JI, Leonard EM, et al. Clinical implications of positive tests for antibodies to human immunodeficiency virus type 1 in asymptomatic blood donors. N Engl J Med. 1989 Oct;321(14):917-24.

10 Doll LS, Petersen LR, White CR, Ward JW; The HIV Blood Donor Study Group. Human immunodeficiency virus type 1 -infected blood donors: behavioral characteristics and reasons for donation. Transfusion. 1991 Oct; 31(8):704-9. 
11 Polizzotto MN, Wood EM, Ingham H, Keller AJ; Australian Red Cross Blood Service Donor and Product Safety Team. Reducing the risk of transfusion-transmissible viral infection through blood donor selection: the Australian experience 2000 through 2006. Transfusion. 2008 Jan;48(1):55-63.

12 Custer B, Kessler D, Vahidnia F, Leparc G, Krysztof DE, Shaz B, et al.; NHLBI Retrovirus Epidemiology Donor Study-II (REDS-II). Risk factors for retrovirus and hepatitis virus infections in accepted blood donors. Transfusion. 2015 May;55(5):1098-107.

13 Goncalez TT, Sabino EC, Murphy EL, Chen S, Chamone DA, McFarland W. Human immunodeficiency virus test-seeking motivation in blood donors, São Paulo, Brazil. Vox Sang. 2006 Apr;90(3):170-6.

14 Gonçalez TT, Sabino EC, Salles NA, de Almeida-Neto C, Mendrone A Jr, Dorlhiac-Laccer PE, et al.; REDS-II International Brazil Study. The impact of simple donor education on donor behavioral deferral and infectious disease rates in São Paulo, Brazil. Transfusion. 2010 Apr;50(4):909-17.

15 de Almeida-Neto C, Goncalez TT, Birch RJ, de Carvalho SM, Capuani L, Leão SC, et al.; NHLBI Retrovirus Epidemiology Donor Study-II (REDS-II) International Component, Brazil. Risk factors for human immunodeficiency virus infection among Brazilian blood donors: a multicentre case-control study using audio computer-assisted structured interviews. Vox Sang. 2013 Aug;105(2): 91-9.

16 Custer B, Sheon N, Siedle-Khan B, Pollack L, Spencer B, Bialkowski W, et al.; NHLBI Recipient Epidemiology and Donor Evaluation Study-III (REDS-III). Blood donor deferral for men who have sex with men: the Blood Donation Rules Opinion Study (Blood DROPS). Transfusion. 2015 Dec;55(12): 2826-34.

17 Grenfell P, Nutland W, McManus S, Datta J, Soldan K, Wellings K. Views and experiences of men who have sex with men on the ban on blood donation: a cross sectional survey with qualitative interviews. BMJ. 2011 Sep; 343:d5604.

18 Moher D, Liberati A, Tetzlaff J, Altman DG; PRISMA Group. Preferred reporting items for systematic reviews and meta-analyses: the PRISMA statement. PLoS Med. 2009 Jul 6(7):e1000097.

19 Guyatt GH, Oxman AD, Vist GE, Kunz R, Falck-Ytter Y, Alonso-Coello P, et al.; GRADE Working Group. GRADE: an emerging consensus on rating quality of evidence and strength of recommendations. BMJ. 2008 Apr;336(7650):924-6

20 Mustafa RA, Santesso N, Brozek J, Akl EA, Walter SD, Norman G, et al. The GRADE approach is reproducible in assessing the quality of evidence of quantitative evidence syntheses. J Clin Epidemiol. 2013 Jul;66(7):736-42; quiz 742.e1-5.

21 GRADEpro GDT: GRADEpro Guideline Development Tool [Software]. McMaster University, 2015 (developed by Evidence Prime, Inc.). Available from: gradepro.org.

22 Silvergleid AJ, Leparc GF, Schmidt PJ. Impact of explicit questions about high-risk activities on donor attitudes and donor deferral patterns. Results in two community blood centers. Transfusion. 1989 May;29(4):362-4.

23 Mayo DJ, Rose AM, Matchett SE, Hoppe PA, Solomon JM, McCurdy KK. Screening potential blood donors at risk for human immunodeficiency virus. Transfusion. 1991 Jun;31(5): 466-74.

24 Gimble JG, Friedman LI. Effects of oral donor questioning about high-risk behaviors for human immunodeficiency virus infection. Transfusion. 1992 Jun;32(5):446-9.

25 Locke SE, Kowaloff HB, Hoff RG, Safran C, Popovsky MA, Cotton DJ, et al. Computerbased interview for screening blood donors for risk of HIV transmission. JAMA. 1992 Sep;268(10):1301-5.

26 Johnson ES, Doll LS, Satten GA, Lenes B, Shafer AW, Kamel H, et al. Direct oral questions to blood donors: the impact on screening for human immunodeficiency virus. Transfusion. 1994 Sep;34(9):769-74.

27 Norris A, Galea G. The impact of the new tickbox questionnaire, and the personal donor interview, on donor deferrals in the East of Scotland. Transfus Med. 2001 Jun;11(3):183-7.

28 Sellors JW, Hayward R, Swanson G, Ali A, Haynes RB, Bourque R, et al. Comparison of deferral rates using a computerized versus written blood donor questionnaire: a randomized, cross-over study [ISRCTN84429599]. BMC Public Health. 2002 Aug; 2(1):14.

29 Katz LM, Cumming PD, Wallace EL, Abrams PS. Audiovisual touch-screen computer-assisted self-interviewing for donor health histories: results from two years experience with the system. Transfusion. 2005 Feb;45(2):17180.

30 Blatyta PF, Custer B, Gonçalez TT, Birch R, Lopes ME, Lopes Ferreira MI, et al.; NHLBI Retrovirus Epidemiology Donor Study-II International Component. Undisclosed human immunodeficiency virus risk factors identified through a computer-based questionnaire program among blood donors in Brazil. Transfusion. 2013 Nov;53(11):2734-43.

31 Turner CF, Ku L, Rogers SM, Lindberg LD, Pleck JH, Sonenstein FL. Adolescent sexual behavior, drug use, and violence: increased reporting with computer survey technology. Science. 1998 May;280(5365):867-73.

32 Islam MM, Topp L, Conigrave KM, van Beek I, Maher L, White A, et al. The reliability of sensitive information provided by injecting drug users in a clinical setting: clinician-administered versus audio computer-assisted self-interviewing (ACASI). AIDS Care. 2012; 24(12):1496-503.

33 U.S. Department of Health and Human Services, Food and Drug Administration, Center for Biologics Evaluation and Research. Guidance for industry: streamlining the donor interview process, recommendations for selfadministered questionnaires. 2003. Available from: https://www.fda.gov/media/70688/ download.

34 U.S. Department of Health and Human Services, Food and Drug Administration. FDA Memorandum to All Registered Blood Establishments: Revised Recommendations for the Prevention of Human Immunodeficiency Vi- rus (HIV) Transmission by Blood and Blood Products. April 23, 1992.

35 U.S. Department of Health and Human Services, Food and Drug Administration, Center for Biologics Evaluation and Research. Revised Recommendations for Reducing the Risk of Human Immunodeficiency Virus Transmission by Blood and Blood Products. 2015. Available from: https://www.fda.gov/ media/92490/download.

36 Higgins JP, Green S, editors. Cochrane Handbook for Systematic Reviews of Interventions Version 5.1.0 [updated March 2011]. The Cochrane Collaboration, 2011. Available from: https://www.handbook.cochrane.org.

37 Weisband S, Kiesler S. Self disclosure on computer forms: meta-analysis and implications. CHI '96 Proceedings of the SIGCHI Conference on Human Factors in Computing Systems. 1996. p. 3-10.

38 Lessler JT, O’Reilly JM. Mode of interview and reporting of sensitive issues: design and implementation of audio computer-assisted self-interviewing. NIDA Res Monogr. 1997; 167:366-82.

39 Kissinger P, Rice J, Farley T, Trim S, Jewitt K, Margavio V, et al. Application of computerassisted interviews to sexual behavior research. Am J Epidemiol. 1999 May;149(10):950-4.

40 Newman JC, Des Jarlais DC, Turner CF, Gribble J, Cooley P, Paone D. The differential effects of face-to-face and computer interview modes. Am J Public Health. 2002 Feb;92(2):294-7.

41 Pluhar E, McDonnell Holstad M, Yeager KA, Denzmore-Nwagbara P, Corkran C, Fielder $\mathrm{B}$, et al. Implementation of audio computerassisted interviewing software in HIV/AIDS research. J Assoc Nurses AIDS Care. 2007 JulAug; 18(4):51-63.

42 O’Brien SF, Ram SS, Vamvakas EC, Goldman M. The Canadian blood donor health assessment questionnaire: lessons from history, application of cognitive science principles, and recommendations for change. Transfus Med Rev. 2007 Jul;21(3):205-22.

43 Des Jarlais DC, Paone D, Milliken J, Turner CF, Miller H, Gribble J, et al. Audio-computer interviewing to measure risk behaviour for HIV among injecting drug users: a quasi-randomised trial. Lancet. 1999 May;353(9165): 1657-61.

44 Rugege-Hakiza SE, Glynn SA, Hutching ST, Bethel J, Nass CC, McEntire RL, et al.; Retrovirus Epidemiology Donor Study. Do blood donors read and understand screening educational materials? Transfusion. 2003 Aug; 43(8):1075-83.

45 Willson S, Miller K, Seem D, Kuehnert MJ. Cognitive evaluation of the AABB Uniform Donor History Questionnaire. Transfusion. 2016 Jun;56(6 Pt 2):1662-7.

46 American Association of Blood Banks (AABB). Final Report of the Task Force to Redesign the Blood Donor Screening Questionnaire, March 2002.

47 Goldman M, Ram SS, Yi QL, O’Brien SF. The Canadian donor health assessment questionnaire: can it be improved? Transfusion. 2006 Dec;46(12):2169-75.

48 Chell K, Davison TE, Masser B, Jensen K. A systematic review of incentives in blood donation. Transfusion. 2018 Jan;58(1):242-54. 